\title{
Tailoring of the resonant mode properties of optical nanocavities in two-dimensional photonic crystal slab waveguides
}

\author{
Oskar Painter ${ }^{1,3}$, Kartik Srinivasan ${ }^{1}$, John D O'Brien ${ }^{2}$, \\ Axel Scherer ${ }^{1}$ and P Daniel Dapkus ${ }^{2}$ \\ ${ }^{1}$ Department of Applied Physics, California Institute of Technology, Pasadena, \\ CA 91125, USA \\ ${ }^{2}$ Department of Electrical Engineering-Electrophysics, University of Southern California, \\ Los Angeles, CA 90089-0271, USA \\ E-mail: opainter@cco.caltech.edu
}

Received 9 August 2001, in final form 28 September 2001

Published 26 October 2001

Online at stacks.iop.org/JOptA/3/S161

\begin{abstract}
Optically thin dielectric slabs, in which a fully etched-through two-dimensional patterning is applied, are used to form high- $Q$ optical cavities with modal volumes approaching the theoretical limit of a cubic half-wavelength. Resonant cavities are formed from local defect regions within the photonic lattice. Simple group theoretical techniques are developed to design cavities which support resonant modes with a particular polarization and radiation pattern. Numerical simulations using the finite-difference time-domain method are then used to study the detailed emission and loss properties of these modes. The cavities are probed spectroscopically through photoluminescence measurements, which when compared with numerical results show the presence of both donor and acceptor type modes. These experimental results show the predictive power of the modest symmetry analysis presented here in describing highly localized defect states within photonic crystals.
\end{abstract}

Keywords: Photonic crystal, microcavity, semiconductor laser, InGaAsP

\section{Introduction}

Planar photonic crystal (PC) waveguide structures [1-3] have been the subject of intense research activity in recent years, as they hold a great deal of promise for use in future generations of high-density lightwave circuits. Significant progress has been made in the study of in-plane waveguides [4-7], coupled resonator-waveguide systems [8$10]$ and add-drop devices [11,12]. One particular area of interest is the study of optical nanocavities in two-dimensional PC slab waveguides, where the strong dispersion of the photonic lattice can be used to trap light in volumes close to the theoretical limit of a cubic half-wavelength [13-16]. Mature fabrication technologies such as planar lithography allow for the precise control of the cavity geometry in these systems [17].

3 To whom correspondence should be addressed.
As a result, the development of basic design methods for PC nanocavities is of considerable importance. In particular, the ability to create optical nanocavities with defect mode resonances of a given polarization, frequency and emission pattern has relevance beyond just optical device technologies, in the development of PC-based systems for more fundamental studies such as cavity-QED explorations of coherent electronphoton interactions.

In this paper, we examine the resonant mode properties of intentionally introduced localized defects within twodimensional PC slab waveguides. The cavity of interest here is composed of an optically thin dielectric slab surrounded by air and patterned with a two-dimensional hexagonal array of holes (figure $1(a)$ ). A defect is created by altering the dielectric constant in a small region of space in the hope of trapping light within the defect region. This can be achieved in practice by varying the radius of the central hole, or by removing a hole 


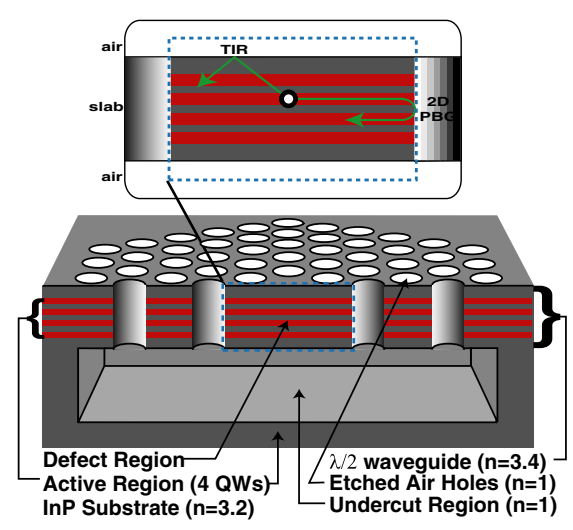

(a)

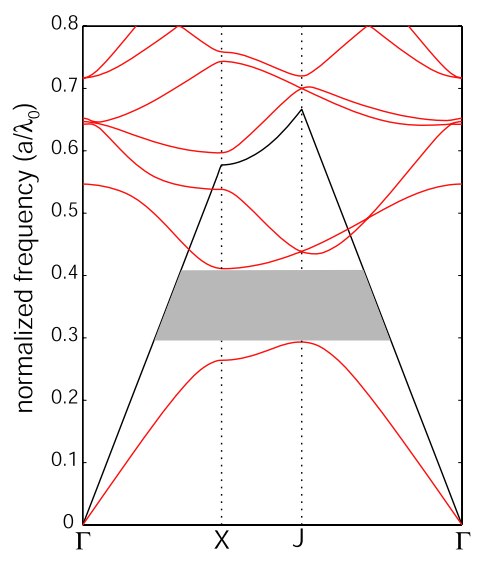

(b)

Figure 1. (a) Illustration of the two-dimensional hexagonal PC slab waveguide structure. $(b)$ Fundamental TE-like (even) guided mode bandstructure $\left(r / a=0.36, n_{\text {slab }}=n_{\text {eff }}=2.65\right)$. The guided mode bandgap extends over a normalized frequency of $0.29-0.41$. The air (cladding) light line is shown as a solid black curve.

entirely and varying the nearest-neighbour holes. By changing the nature of the defect in such a manner, one can profoundly affect the properties of the resonant cavity modes. In particular, the polarization and radiation pattern of the emitted modes can be largely controlled by the design of a suitable defect cavity.

The design and analysis of a given defect cavity begins with a study of the symmetries present in the system. Simple group theoretical techniques are used to produce approximate forms for the resonant modes of the cavity. In addition, the symmetry analysis provides information on the transformation properties of these resonant modes, as well as a scheme for labelling them. The results of the group theory analysis are extended through numerical calculations using the finitedifference time-domain (FDTD) method [14]. FDTD allows for a detailed investigation of the emission characteristics of the PC cavities, including their spectral response, resonant mode field patterns and loss properties.

The following sections detail a study of the optical properties of experimentally realized defect cavities of varying symmetry in a two-dimensional PC slab waveguide. Section 2 outlines the application of simple group theory in producing approximate results describing many of the salient properties of the PC defect cavities. Section 3 presents the results of FDTD simulations, establishing a close correspondence with the group theory analysis while elucidating more detailed properties of the defect mode resonances. In addition, experimental data in the form of spectroscopic probing through photoluminescence (PL) measurements of fabricated devices is given.

\section{Symmetry analysis}

The spatial symmetries within Maxwell's equations are determined by the translation and rotary-reflection symmetries of the dielectric function, $\epsilon(\boldsymbol{r})[18,19]$. As such, group theory can be used to predict and categorize the resonant modes of defects within PC structures. A two-step process is implemented here. The unperturbed slab waveguide modes are first used to generate approximate field patterns for the PC modes at the band-edges defining the guided mode bandgap.
The PC band-edge states are then used as a symmetry basis to generate approximate forms for the localized defect modes lying within the bandgap.

\subsection{Band-edge modes}

The modes of a symmetric slab waveguide, patterned or unpatterned, separate into modes of even or odd parity with respect to a mirror plane in the middle of the dielectric slab. Of interest here are the fundamental guided modes, which, for the slab thicknesses of the devices analysed in section 3, have a wavelength commensurate with the emission band of the quantum wells. This effectively reduces the spatial dimension of the problem from three to two. In the mirror plane of the waveguide, the fundamental even and odd modes can be represented by scalar fields $B_{z}$ and $E_{z}$, respectively.

For the hexagonal photonic lattice of the cavities studied here a bandgap opens up in the frequency spectrum of the fundamental even guided modes, but not in the fundamental odd mode spectrum [14]. For this reason we focus on the fundamental even modes (TE-like) of the slab waveguide, whose magnetic field patterns within the mirror plane of an unpatterned slab are given by $\boldsymbol{B}_{\boldsymbol{k}_{\perp}}\left(\boldsymbol{r}_{\perp}\right)=\hat{z} \mathrm{e}^{-\mathrm{i}\left(\boldsymbol{k}_{\perp} \cdot \boldsymbol{r}_{\perp}\right)}$ for in-plane momentum $\boldsymbol{k}_{\perp}$ and coordinates within the mirror plane $\boldsymbol{r}_{\perp}$. For a two-dimensional PC (with or without vertical waveguiding), the point group symmetry of the hexagonal PC is $D_{6 h}$. Narrowing our scope to TE-like modes of a symmetric slab, the point group symmetry of the system can be effectively reduced to $C_{6 \mathrm{v}}=D_{6 h} / \sigma_{h}$, where the horizontal mirror plane $\left(\sigma_{h}\right)$ lies in the middle of the dielectric slab. A plot of the approximate $^{4}$ in-plane bandstructure for the fundamental TElike guided modes of a half-wavelength thick hexagonally patterned slab waveguide is given in figure1 $(b)$.

Upon patterning the slab waveguide, coupling occurs between waveguide modes with similar unperturbed frequencies and similar propagation constants modulo $G$, where $G$ is a reciprocal lattice vector. The high-symmetry points

4 In this calculation a two-dimensional hexagonal PC with host dielectric constant given by the effective index of the fundamental TE mode of the halfwavelength thick slab is analysed [14]. 


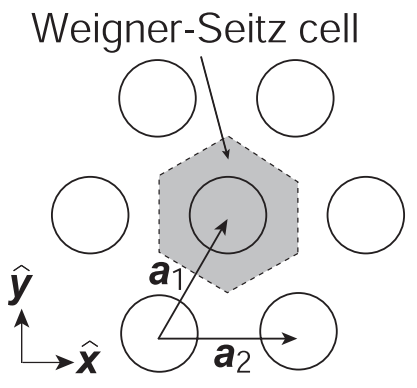

(a)

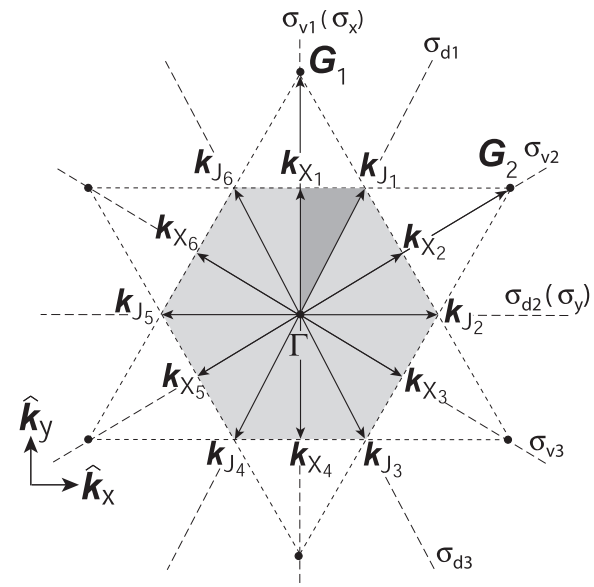

(b)

Figure 2. Illustration of the real and reciprocal spaces of the two-dimensional hexagonal PC. (a) Real space lattice. $\left|a_{1}\right|=\left|a_{2}\right|=a$. (b) Reciprocal space. $\left|\boldsymbol{G}_{1}\right|=\left|\boldsymbol{G}_{2}\right|=4 \pi / \sqrt{3} a,\left|\boldsymbol{k}_{X}\right|=2 \pi / \sqrt{3} a,\left|\boldsymbol{k}_{J}\right|=4 \pi / 3 a$.

of the first Brillouin zone (IBZ) are the six $X$-points $\left(\left\{ \pm(0,1) k_{X}, \pm(\sqrt{3} / 2,1 / 2) k_{X}, \pm(\sqrt{3} / 2,-1 / 2) k_{X}\right\}\right)$, the six $J$-points $\left(\left\{ \pm(1 / 2, \sqrt{3} / 2) k_{J}, \pm(1 / 2,-\sqrt{3} / 2) k_{J}, \pm(1,0) k_{J}\right\}\right)$ and the $\Gamma$-point $=(0,0)$. The first-order bandgap of the hexagonal lattice (see figure $1(b)$ ) is defined from above by the $X$-point ('conduction' band-edge) and below by the $J$-point ('valence' band-edge). The different degenerate $X$ - and degenerate $J$-points in the IBZ are labelled as in figure $2(b)$. The group of the wavevector at the $X$-, $J$ - and $\Gamma$-points is $C_{2 \mathrm{v}}, C_{3 \mathrm{v}}$ and $C_{6 \mathrm{v}}$, respectively. Character tables [20] for these groups are given in table 1.

2.1.1. X-point. From Bloch's theorem we know the eigenmodes at the $X$-point of a hexagonal PC must be the product of a periodic function and a plane wave with momentum $k_{X}$. In the PC structures studied here, we are interested in wavelengths near the first-order bandgap. These wavelengths correspond to the lowest-frequency modes at the $X$-point, which in the unperturbed waveguide have a frequency $\omega_{0}^{X} \approx c\left|\boldsymbol{k}_{X}\right| / n_{\text {eff }}$, where the guided mode has an effective index $n_{\text {eff }}$ and a scalar field within the symmetry plane of the slab waveguide given by $\boldsymbol{B}=\hat{z} \mathrm{e}^{-\mathrm{i} k_{X_{1}} \cdot r_{\perp}}$.

A symmetry basis for the modes of the patterned slab waveguide at the $X$-point (irreducible representations (IRREPs) of the little group at the $X$-point) can be found by applying the symmetry operations of the group of the wavevector $\left(\mathcal{G}_{o k_{X}}=C_{2 \mathrm{v}}\right)$ to the seed vector $\boldsymbol{B}_{k_{X_{1}}}$. In this case, the basis is simply $\left(\boldsymbol{B}_{\boldsymbol{k}_{X_{1}}}, \boldsymbol{B}_{-\boldsymbol{k}_{X_{1}}}\right)$. Projecting this symmetry basis onto the IRREP spaces of $C_{2 v}$ yields

$$
\begin{aligned}
\boldsymbol{B}_{A_{2}}^{X_{1}} & =\hat{z} \cos \left(\boldsymbol{k}_{X_{1}} \cdot \boldsymbol{r}_{\perp}\right), \\
\boldsymbol{B}_{B_{1}}^{X_{1}} & =\hat{z} \sin \left(\boldsymbol{k}_{X_{1}} \cdot \boldsymbol{r}_{\perp}\right),
\end{aligned}
$$

where $A_{2}$ and $B_{1}$ label the IRREP spaces of $C_{2 \mathrm{v}}$ (see table 1). Considering that the dielectric structure, as defined here, has a low-index air hole at the origin, then $\boldsymbol{B}_{A_{2}}^{X_{1}}$ corresponds to the 'valence' band mode and $\boldsymbol{B}_{B_{1}}^{X_{1}}$ to the 'conduction' band mode [21].
In order to fully define the modes at the $X$-point all modes of the star of $k(\star k)$ must be included. The result is the set of degenerate valence band modes,

$$
V B_{A_{2}}^{X}=\hat{z}\left(\begin{array}{c}
\cos \left(\boldsymbol{k}_{X_{1}} \cdot \boldsymbol{r}_{\perp}\right) \\
\cos \left(\boldsymbol{k}_{X_{2}} \cdot \boldsymbol{r}_{\perp}\right) \\
\cos \left(\boldsymbol{k}_{X_{3}} \cdot \boldsymbol{r}_{\perp}\right)
\end{array}\right),
$$

and degenerate conduction band modes,

$$
C B_{B_{1}}^{X}=\hat{z}\left(\begin{array}{c}
\sin \left(\boldsymbol{k}_{X_{1}} \cdot \boldsymbol{r}_{\perp}\right) \\
\sin \left(\boldsymbol{k}_{X_{2}} \cdot \boldsymbol{r}_{\perp}\right) \\
\sin \left(\boldsymbol{k}_{X_{3}} \cdot \boldsymbol{r}_{\perp}\right)
\end{array}\right) .
$$

2.1.2. J-point. A similar procedure may be performed in order to determine approximate forms for the TE-like valence and conduction band modes at the $J$-point of the IBZ. An approximate form for the valence band-edge and conduction band-edge modes at the $J$-point is

$$
\begin{gathered}
V B_{A_{2}^{\prime}}^{J}=\hat{z}\left(\begin{array}{c}
\mathrm{e}^{-\mathrm{i} k_{J_{1}} \cdot r_{\perp}}+\mathrm{e}^{-\mathrm{i} k_{J_{3}} \cdot r_{\perp}}+\mathrm{e}^{-\mathrm{i} k_{J_{5}} \cdot r_{\perp}} \\
\mathrm{e}^{-\mathrm{i} k_{J_{2}} \cdot r_{\perp}}+\mathrm{e}^{-\mathrm{i} k_{J_{4}} \cdot r_{\perp}}+\mathrm{e}^{-\mathrm{i} k_{J_{6}} \cdot r_{\perp}}
\end{array}\right), \\
C B_{E}^{J}=\hat{z}\left(\begin{array}{c}
\mathrm{e}^{-\mathrm{i} k_{J_{1}} \cdot r_{\perp}}+\mathrm{e}^{-\mathrm{i} k_{J_{3}} \cdot r_{\perp}}-2 \mathrm{e}^{-\mathrm{i} k_{J_{5}} \cdot r_{\perp}} \\
\mathrm{e}^{-\mathrm{i} k_{J_{1}} \cdot r_{\perp}}-\mathrm{e}^{-\mathrm{i} k_{J_{3}} \cdot r_{\perp}} \\
\mathrm{e}^{-\mathrm{i} k_{J_{2}} \cdot \boldsymbol{r}_{\perp}}+\mathrm{e}^{-\mathrm{i} k_{J_{4}} \cdot r_{\perp}}-2 \mathrm{e}^{-\mathrm{i} k_{J_{6}} \cdot r_{\perp}} \\
\mathrm{e}^{-\mathrm{i} k_{J_{2}} \cdot r_{\perp}}-\mathrm{e}^{-\mathrm{i} k_{J_{4}} \cdot r_{\perp}}
\end{array}\right),
\end{gathered}
$$

where $A_{2}^{\prime}$ and $E$ are IRREP labels describing how each symmetry basis transforms under $C_{3 \mathrm{v}}$, the point group at the $J$-point.

\subsection{Conduction band donor modes}

By perturbing the dielectric constant in a small region of a periodic PC lattice, one mixes the Bloch modes of the lattice and localized defect modes can form. If the perturbation corresponds to a local increase in the dielectric constant, then the localized modes are formed predominantly from the conduction band modes, specifically the modes at the bandedge. This is a result of the fact that mode frequencies decrease with an increase in the dielectric constant [21]. Thus, the conduction band-edge modes are 'pulled' into the bandgap of 
Table 1. Point group character tables.

\begin{tabular}{c|rrrrrr}
\hline$C_{6 v}$ & $E$ & $C_{2}$ & $2 C_{3}$ & $2 C_{6}$ & $3 \sigma_{d}$ & $3 \sigma_{v}$ \\
\hline$A_{1}^{\prime \prime}$ & 1 & 1 & 1 & 1 & 1 & 1 \\
$A_{2}^{\prime \prime}$ & 1 & 1 & 1 & 1 & -1 & -1 \\
$B_{1}^{\prime \prime}$ & 1 & -1 & 1 & -1 & 1 & -1 \\
$B_{2}^{\prime \prime}$ & 1 & -1 & 1 & -1 & -1 & 1 \\
$E_{1}$ & 2 & -2 & -1 & 1 & 0 & 0 \\
$E_{2}$ & 2 & 2 & -1 & -1 & 0 & 0 \\
\hline$S_{d 1}$ & 3 & -3 & 0 & 0 & 1 & -1 \\
$S_{a 1}$ & 2 & 0 & 2 & 0 & -2 & 0 \\
$S_{a 2}$ & 3 & 3 & 0 & 0 & -1 & -1
\end{tabular}

\begin{tabular}{c|rrrr}
\hline$C_{2 v}$ & $E$ & $C_{2}$ & $\sigma_{x}\left(\sigma_{v 1}\right)$ & $\sigma_{y}\left(\sigma_{d 2}\right)$ \\
\hline$A_{1}$ & 1 & 1 & 1 & 1 \\
$A_{2}$ & 1 & 1 & -1 & -1 \\
$B_{1}$ & 1 & -1 & -1 & 1 \\
$B_{2}$ & 1 & -1 & 1 & -1 \\
\hline$S_{d 1}$ & 3 & -3 & -1 & 1 \\
$S_{a 1}$ & 2 & 0 & 0 & -2 \\
$S_{a 2}$ & 3 & 3 & -1 & -1 \\
\multicolumn{1}{|r}{} & & & \\
\hline$C_{3 v}$ & $E$ & $2 C_{3}$ & $3 \sigma_{d}$ & \\
\hline$A_{1}^{\prime}$ & 1 & 1 & 1 & \\
$A_{2}^{\prime}$ & 1 & 1 & -1 & \\
$E$ & 2 & -1 & 0 &
\end{tabular}

the PC near the defect, resulting in a localized state. This type of localized mode will be termed a donor mode in analogy to the electronic defect states in crystalline materials.

For the hexagonal PC lattice the minimum in the conduction band occurs at the $X$-point (see figure $1(b)$ ). Therefore, the appropriate symmetry basis to use for describing localized donor modes is the degenerate conduction band-edge modes of $C B_{B_{1}}^{X}$. The simplest defect is that of a single missing air hole in the photonic lattice. By removing a hole we have destroyed the translational symmetry of the photonic lattice and are left with its point group symmetry, $C_{6 \mathrm{v}}$.

The matrix representation of the $C B_{B_{1}}^{X}$ basis under the operations of $C_{6 \mathrm{v}}$ is labelled $S_{d 1}$, and its characters are shown in table 1. From the $C_{6 \mathrm{v}}$ character table we find that $S_{d 1}=$ $E_{1} \oplus B_{1}^{\prime \prime}$. Using the projection operators on $C B_{B_{1}}^{X}$, a set of basis functions for $E_{1}$ and $B_{1}^{\prime \prime}$ appears as follows:

$$
\begin{aligned}
\boldsymbol{B}_{B_{1}^{\prime \prime}}^{d 1}= & \hat{z}\left(\sin \left(\boldsymbol{k}_{X_{1}} \cdot \boldsymbol{r}_{\perp}\right)-\sin \left(\boldsymbol{k}_{X_{2}} \cdot \boldsymbol{r}_{\perp}\right)+\sin \left(\boldsymbol{k}_{X_{3}} \cdot \boldsymbol{r}_{\perp}\right)\right), \\
\boldsymbol{B}_{E_{1}, 1}^{d 1}= & \hat{z}\left(2 \sin \left(\boldsymbol{k}_{X_{1}} \cdot \boldsymbol{r}_{\perp}\right)+\sin \left(\boldsymbol{k}_{X_{2}} \cdot \boldsymbol{r}_{\perp}\right)-\sin \left(\boldsymbol{k}_{X_{3}} \cdot \boldsymbol{r}_{\perp}\right)\right), \\
& \boldsymbol{B}_{E_{1}, 2}^{d 1}=\hat{z}\left(\sin \left(\boldsymbol{k}_{X_{2}} \cdot \boldsymbol{r}_{\perp}\right)+\sin \left(\boldsymbol{k}_{X_{3}} \cdot \boldsymbol{r}_{\perp}\right)\right) .
\end{aligned}
$$

The $\left(d 1, B_{1}^{\prime \prime}\right)$ donor mode transforms like a hexapole, whereas the degenerate $\left(d 1, E_{1}\right)$ modes transform as an $(x, y)$-dipole pair.

By introducing defect regions with lower symmetry than the host photonic lattice one is able to remove degeneracies in the localized mode spectrum. The $X$ - and $Y$-split cavities studied below have a defect region with $C_{2 \mathrm{v}}$ symmetry as opposed to the full $C_{6 \mathrm{v}}$ symmetry of the lattice. The effects of this symmetry lowering can be quite easily determined using group theory by looking at the compatibility relations between the IRREPs of the full and reduced symmetry groups:

$$
\begin{gathered}
C_{6 \mathrm{v}} \rightarrow C_{2 \mathrm{v}}, \\
\boldsymbol{B}_{B_{1}^{\prime \prime}}^{d 1} \rightarrow \boldsymbol{B}_{B_{1}}^{d 1,1}, \\
\boldsymbol{B}_{E_{1}, 1}^{d 1} \rightarrow \boldsymbol{B}_{B_{1}}^{d 1,2} \quad(x \text {-dipole }), \\
\boldsymbol{B}_{E_{1}, 2}^{d 1} \rightarrow \boldsymbol{B}_{B_{2}}^{d 1} \quad(y \text {-dipole }) .
\end{gathered}
$$

In the case of the $X$ - and $Y$-split cavities with $C_{2 \mathrm{v}}$ symmetry, group theory predicts the splitting of the degenerate dipolelike modes into $x$ and $y$ dipole-like modes with differing frequencies. This is born out both in the numerical simulations and in the experimental measurements of section 3 below.

\subsection{Valence band acceptor modes}

If the dielectric constant had been reduced in a small region within the photonic lattice, by enlarging an air hole for instance, then instead of pulling the conduction band modes down into the PC bandgap the valence band modes are 'pushed' up into the bandgap. In this case modes localized to the defect region are formed predominantly from mixtures of Bloch modes from the valence band-edge. This type of defect mode is termed an acceptor mode, again in analogy to the electronic states in a crystal. For the hexagonal lattice the maximum of the valence band occurs at the $J$-point (see figure 1(b)). As in the previous section, the obvious symmetry basis used to describe the acceptor modes is the set of degenerate valence band modes at the $J$-point, $V B_{A_{2}^{\prime}}^{J}$.

The simplest type of acceptor defect region consists of an enlargement of a single hole in the photonic lattice. This type of defect maintains the point group symmetry of the lattice, $C_{6 \mathrm{v}}$. The characters of the representation of $V B_{A_{2}^{\prime}}^{J}$ under the $C_{6 \mathrm{v}}$ point symmetry group, which we label $S_{a 1}$, are given in table 1. $S_{a 1}$ decomposes into irreducible blocks $B_{2}^{\prime \prime} \oplus A_{2}^{\prime \prime}$. Using the projection operators, the basis functions of $V B_{A_{2}^{\prime}}^{J}$ are coupled together to form the following localized acceptor modes:

$$
\begin{aligned}
\boldsymbol{B}_{A_{2}^{\prime \prime}}^{a 1} & =\hat{z}\left(\cos \left(\boldsymbol{k}_{J_{1}} \cdot \boldsymbol{r}_{\perp}\right)+\cos \left(\boldsymbol{k}_{J_{3}} \cdot \boldsymbol{r}_{\perp}\right)+\cos \left(\boldsymbol{k}_{J_{5}} \cdot \boldsymbol{r}_{\perp}\right)\right), \\
\boldsymbol{B}_{B_{2}^{\prime \prime}}^{a 1} & =\hat{z}\left(\sin \left(\boldsymbol{k}_{J_{1}} \cdot \boldsymbol{r}_{\perp}\right)+\sin \left(\boldsymbol{k}_{J_{3}} \cdot \boldsymbol{r}_{\perp}\right)+\sin \left(\boldsymbol{k}_{J_{5}} \cdot \boldsymbol{r}_{\perp}\right)\right) .
\end{aligned}
$$

In the $X$ - and $Y$-split cavities with $C_{2 \mathrm{v}}$ symmetry, $\boldsymbol{B}_{A_{2}^{\prime \prime}}^{a 1}$ and $B_{B_{2}^{\prime \prime}}^{a 1}$ transform as $A_{2}$ and $B_{2}$ IRREPs, respectively:

$$
\begin{aligned}
C_{6 \mathrm{v}} & \rightarrow C_{2 \mathrm{v}}, \\
\boldsymbol{B}_{A_{2}^{\prime \prime}}^{a 1} & \rightarrow B_{A_{2}}^{a 1}, \\
\boldsymbol{B}_{B_{2}^{\prime \prime}}^{a 1} & \rightarrow B_{B_{2}}^{a 1} .
\end{aligned}
$$

For defect regions which strongly perturb the photonic lattice it is possible that a larger number of localized defect modes will form than can be described by the limited symmetry basis used above. This is the case for the $Y$-split cavity described below, where the defect region is composed of two enlarged holes and has a relatively deep potential well for acceptor modes. As a result, in the FDTD simulations and the 
PL measurements of the $Y$-split cavity an additional shallow acceptor (SA) type mode $\left(Y-A 2_{0}\right)$, not covered by the $V B_{A_{2}^{\prime}}^{J}$ symmetry basis, is present.

In order to capture more fully the possible defect modes in a deep potential well, the symmetry basis can be expanded in a number of ways. One method would be to modulate the current symmetry basis ( $V B_{A_{2}^{\prime}}^{J}$ ) by a set of envelope functions ${ }^{5}$. However, this would only be applicable in cases where there are two distinct length scales for the defect region and the underlying lattice ${ }^{6}$. Another technique, which could be used in the case of the $Y$-split cavity, is to enlarge the starting symmetry basis by solving for the localized acceptor modes of each enlarged hole within the $Y$-split cavity separately, and then to couple the two basis sets. The symmetry basis in this case includes two copies of the acceptor modes given in equation (8), which are spatially separated by the distance between the two enlarged holes. This method works well for enlarged holes which are significantly displaced so as to be weakly coupled. For the closely spaced enlarged holes of the $Y$-split cavity it is more appropriate to treat the two enlarged holes as a single perturbation of the photonic lattice.

A third method, which will be adopted here, is based upon the observation that for defect regions which provide a deep potential well it is also possible that defect modes will form which are composed of unperturbed PC modes located not just at the edge of the bandgap, but also at other nearby (in frequency) high-symmetry $k$-points within the IBZ. In order to represent these additional localized resonant modes the unperturbed PC modes at the additional high-symmetry $k$-points must be included in the symmetry basis. For the hexagonal lattice the valence band at the $X$-point is close in frequency to the bandgap edge at the $J$-point (see figure $1(b)$ ). The symmetry basis for the $X$-point valence band-edge is the triply degenerate $V B_{A_{2}}^{X}$ basis set. The representation of $V B_{A_{2}}^{X}$ under $C_{6 \mathrm{v}}$, labelled $S_{a 2}$, has the character values shown in table 1 and decomposes into irreducible spaces $E_{2}$ and $A_{2}^{\prime \prime}$. The acceptor type modes formed from the $X$-point valence band modes in a symmetric defect cavity are

$$
\begin{gathered}
\boldsymbol{B}_{A_{2}^{\prime \prime}}^{a 2}=\hat{z}\left(\cos \left(\boldsymbol{k}_{X_{1}} \cdot \boldsymbol{r}_{\perp}\right)+\cos \left(\boldsymbol{k}_{X_{2}} \cdot \boldsymbol{r}_{\perp}\right)+\cos \left(\boldsymbol{k}_{X_{3}} \cdot \boldsymbol{r}_{\perp}\right)\right), \\
\boldsymbol{B}_{E_{2}, 1}^{a 2}=\hat{z}\left(2 \cos \left(\boldsymbol{k}_{X_{1}} \cdot \boldsymbol{r}_{\perp}\right)-\cos \left(\boldsymbol{k}_{X_{2}} \cdot \boldsymbol{r}_{\perp}\right)-\cos \left(\boldsymbol{k}_{X_{3}} \cdot \boldsymbol{r}_{\perp}\right)\right), \\
\boldsymbol{B}_{E_{2}, 2}^{a 2}=\hat{z}\left(\cos \left(\boldsymbol{k}_{X_{2}} \cdot \boldsymbol{r}_{\perp}\right)-\cos \left(\boldsymbol{k}_{X_{3}} \cdot \boldsymbol{r}_{\perp}\right)\right) .
\end{gathered}
$$

The $Y$-split cavity does not have $C_{6 \mathrm{v}}$ symmetry, but rather $C_{2 \mathrm{v}}$ symmetry. This reduction of symmetry causes the $E_{2}$ IRREP space to split into $A_{1} \oplus A_{2}$, and the $A_{2}^{\prime \prime}$ space to transfer

\footnotetext{
5 We have implicitly assumed up to now that the envelope of each of the symmetry basis fields is invariant under all the symmetry operations of the group of the wavevector. This is consistent with assuming that the envelope simply represents exponential decay into the unperturbed regions of the photonic lattice, i.e. contains no nodes representative of higher-order envelope functions.

6 For the highly localized defect cavities studied here the energy associated with the curvature of a higher-order envelope function within the defect region is on the order of the energy associated with the Bloch part of the field.
}

over into an $A_{2}$ IRREP space:

$$
\begin{gathered}
C_{6 \mathrm{v}} \rightarrow C_{2 \mathrm{v}}, \\
\boldsymbol{B}_{A_{2}^{\prime \prime}}^{a 2} \rightarrow \boldsymbol{B}_{A_{2}}^{a 2,1}, \\
\boldsymbol{B}_{E_{2}, 1}^{a 2} \rightarrow \boldsymbol{B}_{A_{2}}^{a 2,2}, \\
\boldsymbol{B}_{E_{2}, 2}^{a 2} \rightarrow \boldsymbol{B}_{A_{1}}^{a 2} .
\end{gathered}
$$

The SA mode $\left(Y-A 2_{0}\right)$ found in the $Y$-split FDTD simulations of the next section transforms as the $A_{2}$ IRREP under $C_{2 \mathrm{v}}$ symmetry operations. The dominant Fourier component within the FDTD-generated field pattern of $Y-A 2_{0}$ is $\boldsymbol{k}_{X_{1}}$, from which we can conclude that this mode is given by $\boldsymbol{B}_{A_{2}}^{a 2,2}$ as opposed to $\boldsymbol{B}_{A_{2}}^{a 2,1}$.

\section{FDTD simulations and photoluminescence measurements}

Expanding upon the symmetry analysis of the defect cavities described above, in this section numerical calculations using the FDTD method [14] are presented. The FDTD simulation results provide information about the resonant frequency, radiation pattern and modal loss of PC defect cavity resonant modes. Moreover, the FDTD calculations establish the effectiveness of the chosen symmetry bases used in the group theoretical analysis of the previous section in describing the defect cavity modes.

The FDTD calculations were performed on a mesh with 20 points per lattice spacing. Excitation of the cavity modes was performed by an initial field $\left(B_{z}\right)$ with a localized Gaussian profile, located in a position of low symmetry so as not to exclude any possible resonant modes. The even modes of the patterned slab waveguide were selected out by using an even mirror symmetry $\left(\sigma_{\mathrm{h}}=+1\right)$ in the middle of the slab waveguide. In order to choose a consistent mode basis (only important for degenerate modes), as well as to reduce computation time, a pair of vertical mirror planes $\left(\sigma_{x}, \sigma_{\mathrm{y}}\right)$ were used to filter out cavity modes according to their projection on the IRREPs of $C_{2 \mathrm{v}}$. Each cavity mode is thus labelled by the $C_{2 \mathrm{v}}$ IRREP by which it transforms and an index corresponding to its energy (frequency) level.

PL measurements performed on microfabricated PC cavities are also presented in conjunction with the FDTD simulations, thus providing a strong tie between theoretical and experimental analyses. For these measurements the PC structures were formed in a waveguide layer containing multiple InGaAsP quantum wells which emit light in the $1500 \mathrm{~nm}$ wavelength band [22]. Optical pumping was provided by a $830 \mathrm{~nm}$ semiconductor laser diode, and the resulting PL was collected from a direction normal to the surface of InGaAsP sample (vertical emission from the planar defect cavities). A more detailed description of the fabrication process and measurement set-up can be found in [18].

\subsection{Symmetric $(S)$ cavity}

The simplest cavity geometry that can be readily implemented consists of a single missing hole (schematically shown in figure $3(a)$ ). We shall refer to this cavity as a symmetric or 


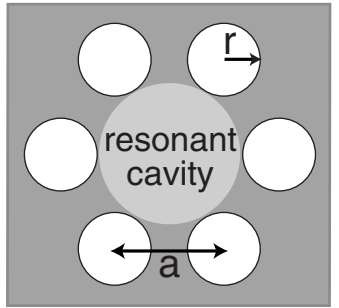

(a)

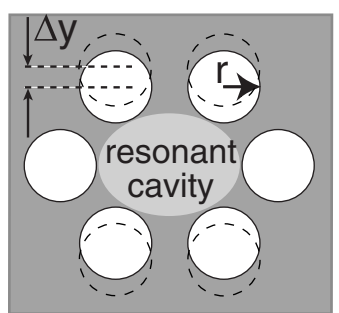

(b)

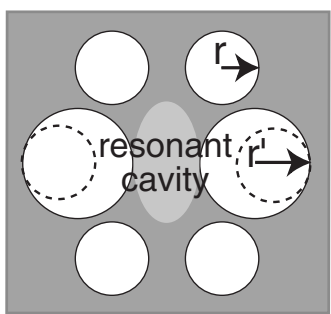

(c)

Figure 3. PC defect cavity geometries. (a) $S$-cavity. (b) $X$-split cavity. (c) $Y$-split cavity.

$S$-cavity as it retains the full point group symmetry of the hexagonal lattice $\left(C_{6 \mathrm{v}}\right)$. An FDTD-simulated spectrum of a defect cavity with a central missing hole and a linear grade ${ }^{7}$ in $r / a$ (from the centre outwards) of $0.38-0.34$ is plotted in figure 4 as a dashed curve. The spectrum is plotted versus normalized frequency, $\omega_{n}=a / \lambda_{0}$, where $a$ is the lattice spacing and $\lambda_{0}$ is the free-space wavelength. A normalized slab thickness, $d / a$, of 0.41 was used in the simulated structures to be consistent with the fabricated devices. To reduce computation time, the number of mirror periods $(p)$ surrounding the central missing hole was limited to five in the simulations, save for the more extended modes for which cavities with eight periods were also simulated in order to more accurately estimate the modal losses present in the fabricated devices (see table 2).

In figure 4 , there appear to be two distinct resonance peaks within the guided mode bandgap of the TE-like modes. Performing a mode filter [14] using the $C_{2 \mathrm{v}}$ mirror planes, we find that each resonance peak contains two different modes, yielding a total of four different localized modes whose magnetic field patterns within the mirror symmetry plane of the slab are shown in table 2 . The two resonant modes (accidentally degenerate) associated with the peak near the valence band-edge correspond to SA modes which transform as the $A_{2}^{\prime \prime}$ and $B_{2}^{\prime \prime}$ IRREPs of $C_{6 \mathrm{v}}$, and have the same dominant in-plane Fourier components as $\boldsymbol{B}_{A_{2}^{\prime \prime}}^{a 1}$ and $\boldsymbol{B}_{B_{2}^{\prime \prime}}^{a 1}$ of the symmetry analysis in section 2 . The addition of these $\mathrm{SA}$ modes is a result of the linear grading in hole radius, which forms a potential well for acceptor-type modes. Of particular interest is the strongly localized pair of degenerate deep donor (DD) dipolelike modes near the centre of the bandgap. From the plots of the electric field intensity of the $x$ - and $y$-dipole modes shown in figures 5(a) and $(b)$, we see that the fundamental $k$-components

\footnotetext{
7 As a result of nonidealities in the fabrication process [22], the air holes near the centre of the cavity are larger than those at the perimeter in the fabricated devices. A linear grading of the hole radius of $10 \%$ is quite common.
}

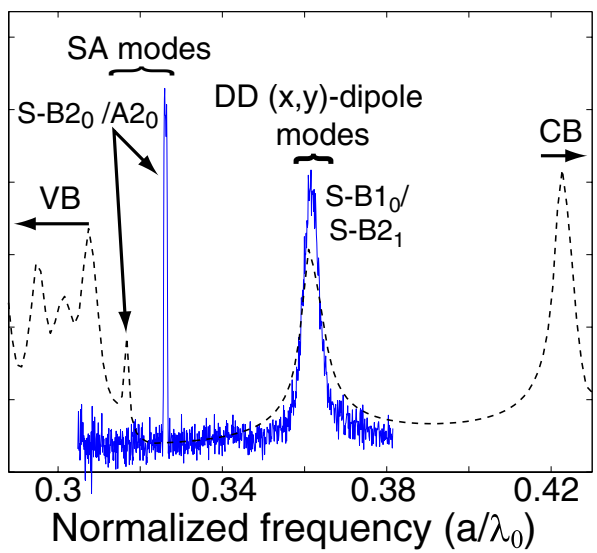

Figure 4. FDTD and PL spectra of an $S$-type defect cavity with $a=515 \mathrm{~nm}, r / a=0.36$ nominally (graded centre outwards from $0.38-0.34), n_{\text {slab }}=3.4$ and $d / a=0.409$. FDTD simulation results are shown as a dashed curve.

of the $x$ and $y$ dipole-like modes correspond nicely with the approximate field patterns predicted by the symmetry analysis. $\boldsymbol{B}_{E_{1}, 1}^{d 1}$ represents the $x$-dipole mode and $\boldsymbol{B}_{E_{1}, 2}^{d 1}$ the $y$-dipole mode. Even the subtle difference in the in-plane radiation pattern of the $(x, y)$-dipole modes as calculated numerically using FDTD is contained within the symmetry analysis as can be seen by the lack of a third standing wave component in the $y$-dipole $\left(\boldsymbol{B}_{E_{1}, 2}^{d 1}\right)$ mode.

A PL spectrum from an $S$-defect cavity with $a=515 \mathrm{~nm}$, $r / a \approx 0.36$ and $d / a=0.41$ is shown overlaid upon the FDTD simulation in figure 4 . The emission from the $S$-cavity also shows the presence of two dominant peaks, one very close to the DD peak and one close to the SA peak. Owing to the small scale of the PC defect cavities, the field patterns of the DD modes strongly resemble that of an oscillating electric dipole. A vector plot of the $\boldsymbol{E}$-field of the $x$ - and $y$-dipole modes in the plane of the slab is shown in figures 5(c) and $(d)$. Polarization measurements of the DD peak [23] confirm that the emission is polarized predominantly along two orthogonal directions consistent with the $x$ - and $y$-dipole directions. The experimental determination of the absolute frequency of the DD dipole modes provides a reference point from which to classify the rest of the cavity modes, and also provides a measure of the accuracy of the FDTD calculations.

A list of properties of the two SA and two DD localized defect modes is given in table 2 . The numerically calculated losses of each cavity mode are represented by effective inplane and out-of-plane quality factors [14], $Q_{\|}$and $Q_{\perp}$, respectively. The effective mode volume, $V_{\text {eff }}$, is calculated from an estimate of the full-width at half-maximum value of the electric field energy density in each direction [24], and is given in units of cubic half-wavelengths. As a result of the large porosity of the PC obtained during the fabrication process [22], FDTD simulations predict a rather large vertical diffraction loss (small $Q_{\perp}$ ) for the highly localized dipolelike modes. In contrast, the unintentionally introduced linear grade in hole radius provides sufficient in-plane localization to produce high- $Q$ SA modes. For the fabricated defect cavities with eight periods of the PC mirror $(p=8)$, the quality factor for the $S-B 2_{0}$ mode is theoretically estimated to be as high as 7500 , limited by radiative losses in the plane of the photonic 
Table 2. Characteristics and magnetic field amplitude patterns of the resonant modes in a symmetric cavity with $r / a$ linearly graded from $0.38-0.34\left(d / a=0.409, n_{\text {slab }}=3.4, p=5\right)$.

\begin{tabular}{|c|c|c|c|c|c|c|}
\hline A2 $(-1,-1)$ & \multirow{2}{*}{\multicolumn{2}{|c|}{$\frac{\mathrm{B1}(-1,+1)}{\mathrm{S}-\mathrm{B} 1_{0}}$}} & \multicolumn{4}{|c|}{ B2 $(+1,-1)$} \\
\hline $\mathrm{S}-\mathrm{A} 2_{0}$ & & & \multicolumn{3}{|c|}{$\mathrm{S}-\mathrm{B} 2{ }_{0}$} & $\mathrm{~S}-\mathrm{B} 21$ \\
\hline $\begin{array}{c}0000000 \\
00000000 \\
0000000000 \\
000000000 \\
0000000 \\
0000000\end{array}$ & \multicolumn{2}{|c|}{ 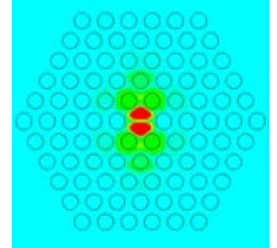 } & \multicolumn{3}{|c|}{$\begin{array}{c}000000 \\
0000000 \\
00000000 \\
000000000100 \\
0000000000 \\
0000000000 \\
000000000 \\
000000000 \\
0000000 \\
000000\end{array}$} & 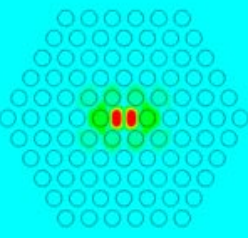 \\
\hline Label & Grp. & $\left(\sigma_{\mathbf{x}}, \sigma_{\mathbf{y}}\right)$ & $\omega_{\mathbf{n}}$ & $\mathbf{Q}_{\|}$ & $\mathbf{Q}$ & $V_{\text {eff }}$ \\
\hline $\mathrm{S}-\mathrm{A} 2_{0}\left(\mathbf{B}_{A_{2}^{\prime \prime}}^{a 1}\right)$ & $\mathrm{SA}$ & $(-,-)$ & 0.320 & $4800^{\mathrm{a}}$ & $1.0 \mathrm{e} 4^{\mathrm{a}}$ & 17.6 \\
\hline $\mathrm{S}-\mathrm{B} 1_{0}\left(\mathbf{B}_{E_{1}, 1}^{d 1}\right)$ & DD & dipole $(\mathbf{x})$ & 0.361 & 1180 & 150 & 2.7 \\
\hline $\mathrm{S}-\mathrm{B} 2_{0}\left(\mathbf{B}_{B_{0}^{\prime \prime}}^{a 1}\right)$ & $\mathrm{SA}$ & $(+,-)$ & 0.322 & $8800^{\mathrm{a}}$ & $6.4 \mathrm{e} 4^{\mathrm{a}}$ & 12 \\
\hline $\mathrm{S}-\mathrm{B} 2_{1}\left(\mathbf{B}_{E_{1}, 2}^{d 1}\right)$ & DD & dipole $(\mathbf{y})$ & 0.360 & 1370 & 180 & 2.7 \\
\hline
\end{tabular}

a $p=8$.

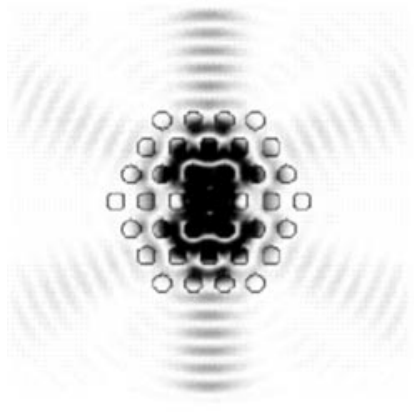

(a)

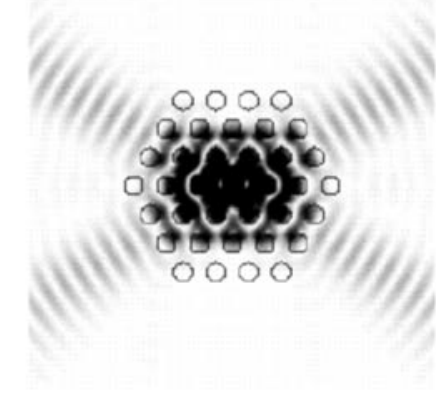

(b)

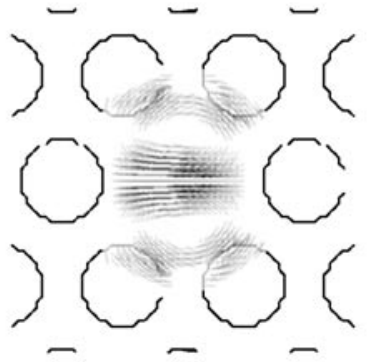

(c)

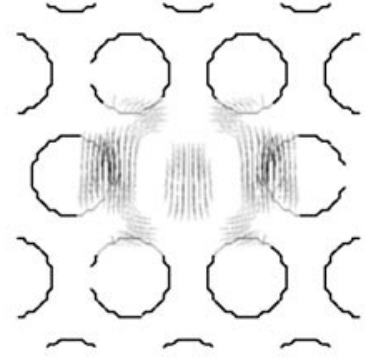

(d)

Figure 5. In-plane radiation losses (electric field amplitude saturated) of the $x$ - and $y$-dipole modes (degenerate case) are shown in (a) and $(b)$, respectively. In $(c)$ and $(d)$ the vector plots of the electric field of the $(x, y)$-dipole modes in the middle of the slab waveguide are shown.

lattice. It is for this reason that room-temperature lasing in $S$-defect cavities is limited to the SA mode peak [18], and reduced temperatures are required in order for the DD dipolelike modes to lase (see section 3.3).

\section{2. $X$-split $(X)$ cavity}

Another type of defect cavity that was fabricated and tested, referred to as the $X$-split cavity, is shown in figure $3(b)$. In this cavity the four air holes on the top and bottom of the central missing hole are moved inwards a distance $\Delta y$, reducing the defect symmetry from $C_{6 \mathrm{v}}$ to $C_{2 \mathrm{v}}$. In the fabricated structures $\Delta y \sim 0.05 a \sim 25 \mathrm{~nm}$. An FDTD spectrum of an $X$-split cavity with $\Delta y$ matching that of the fabricated devices is plotted in figure 6. The magnetic field amplitudes of the different resonant modes found in the FDTD spectrum are shown in table 3 . The DD $(x, y)$-dipole modes are seen to split, as expected from the symmetry analysis of the previous section, with the $x$-dipole being higher in frequency. The geometry of the cavity also introduces two SA modes, $X-A 2_{0}$ and $X-B 2_{0}$. These are the same modes as those found in the $S$-cavity in which the linear grade in hole size created a potential well for

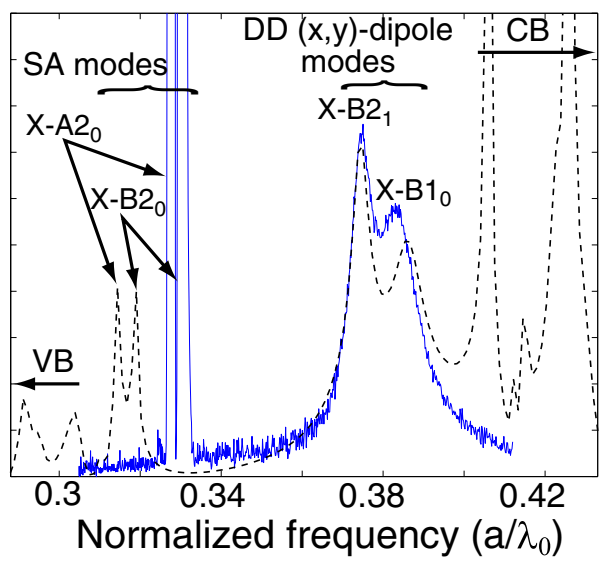

Figure 6. PL and FDTD spectra showing the resonant modes of a $X$-split defect cavity with $r / a=0.38-0.34, d / a=0.409$,

$n_{\text {slab }}=3.4$ and $\Delta y=0.05 a$. FDTD simulation results are shown as a dashed curve.

additional acceptor-type modes. As such, these modes have the same dominant in-plane Fourier components as $\boldsymbol{B}_{A_{2}}^{a 1}$ and $\boldsymbol{B}_{B_{2}}^{a 1}$ of section 2 . 
Table 3. Characteristics and magnetic field (amplitude) patterns of the resonant modes in an $X$-split cavity $(r / a=0.38-0.34, d / a=0.409$, $\left.n_{\text {slab }}=3.4, \Delta y=0.05 a, p=5\right)$.

\begin{tabular}{|c|c|c|c|c|c|c|}
\hline A2 $(-,-)$ & \multirow{2}{*}{\multicolumn{2}{|c|}{$\frac{\mathrm{B} 1(-,+)}{\mathrm{X}-\mathrm{B} 1_{0}}$}} & \multicolumn{4}{|c|}{ B2 $(+,-)$} \\
\hline $\mathrm{X}-\mathrm{A} 2_{0}$ & & & \multicolumn{3}{|c|}{$\mathrm{X}-\mathrm{B} 2_{0}$} & $\mathrm{X}-\mathrm{B} 2_{1}$ \\
\hline 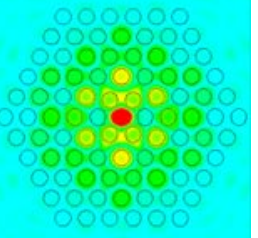 & \multicolumn{2}{|c|}{ 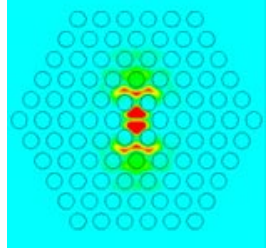 } & \multicolumn{3}{|c|}{ 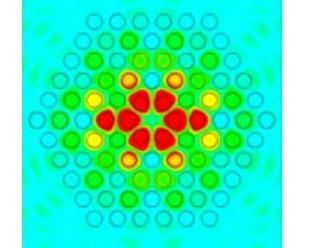 } & 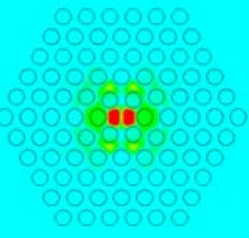 \\
\hline Label & Grp. & $\left(\sigma_{x}, \sigma_{y}\right)$ & $\omega_{\mathbf{n}}$ & $\mathbf{Q}_{\|}$ & $\mathbf{Q}_{\perp}$ & $V_{\text {eff }}$ \\
\hline $\mathrm{X}-\mathrm{A} 2_{0}\left(\mathbf{B}_{A_{2}}^{a 1}\right)$ & $\mathrm{SA}$ & $(-,-)$ & 0.316 & $962^{\mathrm{a}}$ & $3680^{\mathrm{a}}$ & 9.7 \\
\hline $\mathrm{X}-\mathrm{B} 1_{0}\left(\mathbf{B}_{B_{1}}^{d 1,2}\right)$ & DD & dipole (x) & 0.385 & 400 & 60 & 3.2 \\
\hline $\mathrm{X}-\mathrm{B} 2_{0}\left(\mathbf{B}_{B_{2}}^{a 1}\right)$ & $\mathrm{SA}$ & $(+,-)$ & 0.319 & $3970^{\mathrm{a}}$ & $1740^{\mathrm{a}}$ & 5.5 \\
\hline $\mathrm{X}-\mathrm{B} 2_{1}\left(\mathbf{B}_{B_{2}}^{d 1}\right)$ & $\mathrm{DD}$ & dipole $(\mathbf{y})$ & 0.374 & 650 & 100 & 2.5 \\
\hline
\end{tabular}

Figure 6 also contains a PL spectrum for an $X$-split cavity $(r / a=0.38-0.34, d / a=0.409, \Delta y=0.05 a)$ overlaid upon the FDTD spectrum. The PL spectrum shows the presence of two pairs of resonance peaks. The SA and DD peaks of the $S$-cavity have each split into two distinct resonances. The positions of these peaks correspond well with those of the FDTD simulation, and allow for their identification (see table 3 ) using the nomenclature developed in section 2. This classification is further supported by polarization measurements of the DD modes [23]. These measurements show that the two modes are highly polarized along orthogonal directions, with the longer-wavelength peak identified as the $y$-dipole mode and the shorter-wavelength peak as the $x$-dipole mode.

FDTD calculated properties of the SA and DD resonant modes of the $X$-split cavity are listed in table 3. As in the $S$-cavity the DD dipole-like modes are seen to be highly localized; however, the vertical diffraction loss suffered by the dipole-like modes is much more severe in the case of the $X$-split cavity, especially so for the $x$-dipole mode. This can be seen in the PL spectrum of the $X$-split cavity, in which the higherfrequency DD peak is significantly broader than its lowerfrequency partner. Room-temperature lasing (pulsed) was limited to the $X-A 2_{0}$ and $X-B 2_{0}$ SA modes. Measurements of the threshold pump power of each SA mode from a large array of devices showed a consistently lower threshold value for the higher-frequency $X-B 2_{0}$ mode [18], in agreement with its higher estimated $Q$-value given in table 3 .

\subsection{Y-split $(Y)$ cavity}

In the $Y$-split cavity, illustrated in figure $3(c)$, the nearestneighbour holes on both sides of the central missing hole along the $\Gamma J$-direction are enlarged and moved slightly inwards. The degree of splitting is measured by $r^{\prime} / r$, the scaling factor of the enlarged holes. The cavities studied here use hole enlargements which result in a much stronger perturbation of the cavity than in the $X$-split case. A FDTD simulation showing the mode spectrum of a $Y$-split cavity with the two

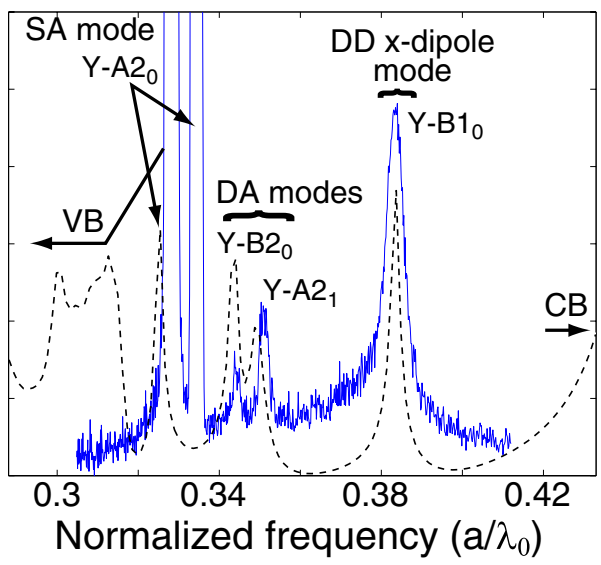

Figure 7. PL and FDTD spectra of the resonant modes in a $Y$-split defect cavity with $r / a=0.38-0.34, r^{\prime} / a=0.51, d / a=0.409$ and $n_{\text {slab }}=3.4$. FDTD simulation results are shown as a dashed curve.

nearest-neighbour holes enlarged by $r^{\prime} / r=1.5$ is shown in figure 7. The magnetic field amplitudes of the various localized defect modes of the $Y$-split cavity are given in table 4. There are now at least four different localized modes within the photonic bandgap. The two enlarged holes act as centres for acceptor modes and give rise to two deep acceptor (DA) modes in the spectrum. These DA modes are labelled as $Y-A 2_{1}$ and $Y-B 2_{0}$, and correspond to the SA modes of the $S$ - and $X$-split cavities. The strength of the perturbation to the photonic lattice produces an additional SA mode as well. As noted in 2, this mode has the same dominant in-plane Fourier components as $\boldsymbol{B}_{A_{2}}^{a 2,2}$ of the symmetry analysis. We further note that the splitting of the $(x, y)$-dipole modes is so strong in this case that the $y$-dipole mode is completely pushed out of the bandgap and only the $x$-dipole mode remains.

The PL spectrum for a $Y$-split cavity $(r / a=0.38-0.34$, $\left.r^{\prime} / a=0.51, d / a=0.409\right)$, shown with the FDTD spectrum in figure 7 confirms many of the predictions made by the group theory and FDTD analyses. In particular, we note the presence of an SA peak, two DA peaks and a single DD peak. 
Tailoring of the resonant mode properties of optical nanocavities in two-dimensional photonic crystal slab waveguides

Table 4. Characteristics and magnetic field (amplitude) patterns of the resonant modes in a $Y$-split cavity $\left(r / a=0.38-0.34, r^{\prime} / a=0.51\right.$, $\left.d / a=0.409, n_{\text {slab }}=3.4, p=8\right)$.

\begin{tabular}{|c|c|c|c|c|c|c|}
\hline \multicolumn{3}{|c|}{ A2 $(-,-)$} & \multirow{2}{*}{\multicolumn{3}{|c|}{$\frac{\mathrm{B} 1(-,+)}{\mathrm{Y}-\mathrm{B} 1_{0}}$}} & \multirow{2}{*}{$\frac{\mathrm{B} 2(+,-)}{\mathrm{Y}-\mathrm{B} 2_{0}}$} \\
\hline $\mathrm{Y}-\mathrm{A} 2_{0}$ & & $\mathrm{r}-\mathrm{A} 2_{1}$ & & & & \\
\hline 28 & & 8080 & \multicolumn{3}{|c|}{$88^{\circ}$} & $38^{\circ}$ \\
\hline Label & Grp. & $\left(\sigma_{x}, \sigma_{y}\right)$ & $\omega_{\mathbf{n}}$ & $\mathbf{Q}_{\|}$ & $\mathbf{Q}_{\perp}$ & $V_{\text {eff }}$ \\
\hline $\mathrm{Y}-\mathrm{A} 2_{0}\left(\mathbf{B}_{A_{2}}^{a 2,2}\right)$ & $\mathrm{SA}$ & $(-,-)$ & 0.326 & 2140 & 350 & 2.8 \\
\hline $\mathrm{Y}-\mathrm{A} 2_{1}\left(\mathbf{B}_{A_{2}}^{a 1}\right)$ & $\mathrm{DA}$ & $(-,-)$ & 0.350 & 690 & 130 & 2.2 \\
\hline $\mathrm{Y}-\mathrm{B} 1_{0}\left(\mathbf{B}_{B_{1}}^{d 1,2}\right)$ & $\mathrm{DD}$ & dipole $(\mathbf{x})$ & 0.384 & 2110 & 235 & 2.8 \\
\hline $\mathrm{Y}-\mathrm{B} 2_{0}\left(\mathbf{B}_{B_{2}}^{a 1}\right)$ & $\mathrm{DA}$ & $(+,-)$ & 0.344 & 2170 & 230 & 2.9 \\
\hline
\end{tabular}

Polarization measurements of this DD mode [23] show it to be strongly polarized in a direction corresponding to the $x$-dipole mode. Table 4 also contains a list of the FDTD-calculated properties of the $Y$-split cavity defect modes. It is interesting to note that the DA modes are as well localized as the DD dipole-like mode. The low $Q$-values of the DA modes and the $x$-dipole mode are due to the large size of the splitting holes. Room-temperature (pulsed) lasing was observed from all but the DD $x$-dipole mode in the $Y$-split cavities [18]. At reduced temperatures $(T<150 \mathrm{~K})$, it was possible to obtain pulsed lasing action of the $x$-dipole mode [16]. As the DA modes have similar $Q$-values to that of the $x$-dipole mode, it is suspected that the difficulty in obtaining lasing from the DD mode may have more to do with the misalignment of the gain spectrum with the resonance wavelength of the defect cavities fabricated and tested in this experiment than with the modal loss.

\section{Summary}

The optical properties of defect cavities in two-dimensional PC slab waveguides have been examined analytically through group theoretical methods, numerically through FDTD calculations and experimentally through PL spectroscopy of fabricated devices. The simple group theory analysis provides significant predictive power by using symmetry arguments to describe the approximate behaviour of a given device, while the FDTD simulations uncover more detailed, quantitative information that complements the group theory results and can be directly compared with experiment. The combination of these two methods results in a highly descriptive model for the behaviour of the defect modes of PC cavities of varying symmetry. Experimental characterization of fabricated structures largely confirms the predictions of this model, and this close correspondence illustrates the degree to which the emission properties of the resonant modes of these systems can be specified by a careful design that utilizes the techniques described in this paper.

\section{Acknowledgments}

The authors would like to thank In Kim and Denis Tishinin for the growth of the InGaAsP material used to create the PC cavities. O Painter would like to thank Kerry Vahala for many fruitful discussions regarding group theory. K Srinivasan thanks the Hertz Foundation for its financial support.

\section{References}

[1] Atkin D M, Russell P S J, Birks T A and Roberts P J 1996 Photonic band structure of guided Bloch modes in high index films fully etched through with periodic microstructure J. Mod. Opt. $\mathbf{4 3}$ 1035-53

[2] Russell P S J, Atkin D M and Birks T A 1996 Microcavities and Photonic Bandgaps (Dordrecht: Kluwer) pp 203-18

[3] Johnson S G, Fan S, Villeneuve P R, Joannopoulos J D and Kolodziejaki L A 1999 Guided modes in photonic crystal slabs Phys. Rev. B 60 5751-8

[4] Mekis A, Chen J C, Kurland I, Fan S, Villeneuve P R and Joannopoulos J D 1996 High transmission through sharp bends in photonic crystal waveguides Phys. Rev. Lett. 77 3787-90

[5] Chow E, Lin S.Y, Wendt J R, Johnson S G and Joannopoulos J D 2001 Quantitative analysis of bending efficiency in photonic-crystal waveguide bends at $\lambda=1.55 \mu \mathrm{m}$ wavelengths Opt. Lett. 26 286-8

[6] Baba T, Fukaya N and Yonekura J 1999 Observation of light propagation in photonic crystal optical waveguides with bends IEE Electron. Lett. 35 654-5

[7] Lončar M, Doll T, Vučković J and Scherer A 2000 Design and fabrication of silicon photonic crystal optical waveguides $J$. Lightwave Technol. 18 1402-11

[8] Yariv A, Xu Y, Lee R K and Scherer A 1999 Coupled-resonator optical waveguide: a proposal and analysis Opt. Lett. 24 711-13

[9] Olivier S, Smith C, Rattier M, Benisty H, Weisbuch C, Krauss T, Houdré R and Oesterlé U 2001 Miniband transmission in a photonic crystal coupled-resonator optical waveguide Opt. Lett. 26 1019-21

[10] Smith C, De la Rue R, Rattier M, Olivier S, Benisty H, Weisbuch C, Krauss T, Oesterlé U and Houdré R 2001 Coupled guide and cavity in a two-dimensional photonic crystal Appl. Phys. Lett. 78 1487-9

[11] Fan S, Villeneuve P R and Joannopoulos J D 1998 Channel drop filters in photonic crystals Opt. Express 3 4-11 
[12] Noda S, Chutinan A and Imada M 2000 Trapping and emission of photons by a single defect in a photonic bandgap structure Nature 407 608-10

[13] Foresi J S, Villeneuve P R, Ferrera J, Thoen E R, Steinmeyer G, Fan S, Joannopoulos J D, Kimerling L C, Smith H I and Ippen E P 1997 Photonic-bandgap microcavities in optical waveguides Nature 390 143-5

[14] Painter O, Vučković J and Scherer A 1999 Defect modes of a two-dimensional photonic crystal in an optically thin dielectric slab J. Opt. Soc. Am. B 16 275-85

[15] Coccioli R, Boroditsky M, Kim K W, Rahmat-Samii Y and Yablonovitch E 1998 Smallest possible electromagnetic mode volume in a dielectric cavity IEE Proc. Optoelectron 145 391-7

[16] Painter O, Lee R K, Yariv A, Scherer A, O’Brien J D, Dapkus P D and Kim I 1999 Two-dimensional photonic band-gap defect mode laser Science 284 1819-24

[17] Painter O, Husain A, Scherer A, Lee P T, Kim I, O’Brien J D and Dapkus P D 2000 Lithographic tuning of a two-dimensional photonic crystal laser array IEEE Photon. Technol. Lett. 12 1126-8

[18] Painter O J 2001 PhD Thesis California Institute of Technology

[19] Sakoda K 1995 Symmetry, degeneracy, and uncoupled modes in two-dimensional photonic crystals Phys. Rev. B 52 7982-6

[20] Tinkham M 1964 Group Theory and Quantum Mechanics (Int. Series in Pure and Applied Physics) (New York: McGraw-Hill)

[21] Joannopoulos J D, Meade R D and Winn J N 1995 Photonic Crystals (Princeton, NJ: Princeton University Press)

[22] Painter O J, Husain A, Scherer A, O’Brien J D, Kim I and Dapkus P D 1999 Room temperature photonic crystal defect lasers at near-infrared wavelengths in InGaAsP $J$. Lightwave Technol. 17 2082-8

[23] Painter O and Srinivasan K 2001 Opt. Lett. submitted

[24] Villeneuve P R, Fan S, Johnson S G and Joannopoulos J D 1998 Three-dimensional photon confinement in photonic crystals of low-dimensional periodicity IEE Proc. Optoelectron. $145384-90$ 